\title{
Expression of fatty-acid-handling proteins in human adipose tissue in relation to obesity and insulin resistance
}

\author{
K. Gertow ${ }^{1}$ K. H. Pietiläinen ${ }^{2,3,4}$ • H. Yki-Järvinen ${ }^{4}$ J. Kaprio ${ }^{2,5}$ • A. Rissanen ${ }^{3}$ P. Eriksson ${ }^{1}$ A. Hamsten ${ }^{1}$ • \\ R. M. Fisher ${ }^{1}$ \\ ${ }^{1}$ Atherosclerosis Research Unit. King Gustaf V Research Institute, Karolinska Institutet, Karolinska Hospital Building M1, \\ Stockholm, Sweden \\ ${ }^{2}$ Finnish Twin Research, Department of Public Health, University of Helsinki, Finland \\ 3 Obesity Research Unit, Department of Psychiatry, Helsinki University Central Hospital, Finland \\ ${ }^{4}$ Department of Medicine, Division of Diabetes, University of Helsinki, Finland \\ 5 Department of Mental Health and Alcohol Research, National Public Health Institute, and Department of Public Health, \\ University of Helsinki, Finland
}

\section{Abstract}

Aims/hypothesis. Protein-mediated trans-membrane and intracellular fatty acid trafficking are becoming increasingly recognised as biochemically and physiologically important concepts. Obesity and insulin resistance are polygenic disorders, heavily influenced by environmental and life-style factors, and are virtually always associated with disturbed fatty acid metabolism in adipose and other tissues. The aim of this study was to investigate mRNA expression levels of fatty-acid-handling proteins in adipose tissue in relation to markers of genetic and acquired obesity and insulin resistance.

Methods. We quantified mRNA expression of subcutaneous adipose tissue fatty-acid-handling proteins (ALBP, KLBP, FATP1, FATP4, CD36, ACS1) in 17 monozygotic twin-pairs with a range of intra-pair differences $(\Delta)$ in BMI and detailed measures of obesity and insulin resistance, allowing influences of genetic and non-genetic factors to be distinguished.

Results. In acquired obesity FATP4 expression was up-regulated independently of genetic background ( $\Delta$ FATP4 versus $\Delta \mathrm{BMI} ; r=0.50, p=0.04 ; \Delta$ FATP4 ver- sus $\Delta$ body fat; $r=0.59, p=0.01)$. Similarly, CD36 and FATP1 expression correlated with acquired differences in HDL cholesterol and non-esterified fatty acid concentrations respectively. Moreover, FATP4 and CD36 expression levels correlated with measures of obesity and insulin resistance that are influenced by both genetic and non-genetic factors (FATP4 versus BMI: $r=0.53, p=0.0001$; FATP4 versus body fat: $r=0.51, p=0.002$; FATP4 versus homeostasis model assessment [HOMA]: $r=0.49, p=0.001$; CD36 versus BMI: $r=0.50, p=0.02$; CD36 versus body fat: $r=0.63$, $p=0.001$; CD36 versus HOMA: $r=0.34, p=0.06$ ).

Conclusions/interpretation. These findings indicate that expression of specific adipose tissue fatty-acidhandling proteins is related to obesity and insulin resistance, and that, in particular, FATP4 plays a role in acquired obesity. Our results suggest that facilitated fatty acid trafficking is a physiologically and pathologically relevant phenomenon in man.

Keywords Adipose tissue - Expression · Fatty-acid-handling protein $\cdot$ Insulin resistance $\cdot$ Obesity $\cdot$ Twin study
Received: 19 December 2003 / Accepted: 3 February 2004

Published online: 28 May 2004

C) Springer-Verlag 2004

R. M. Fisher $(\bullet)$

Atherosclerosis Research Unit. King Gustaf V Research Institute, Karolinska Institutet, Karolinska Hospital Building M1,

17176 Stockholm, Sweden

E-mail: rachel.fisher@medks.ki.se

Tel.: +46-8-51773245, Fax: +46-8-311298

Abbreviations: ACS, acyl-CoA synthase - ALBP, adipocyte lipid-binding protein . FABP, fatty-acid-binding protein .

FATP, fatty acid transport protein $\cdot$ HOMA, homeostasis model assessment $\cdot$ KLBP, keratinocyte lipid-binding protein $\cdot$ MZ, monozygotic $\cdot$ TBP, TATA-box binding protein $\cdot$ TG, triglyceride

\section{Introduction}

Perturbations of fatty acid metabolism in adipose tissue and other tissues are implicated in the development of obesity and insulin resistance. Increased plasma NEFA concentrations, which are a hallmark of obesity and insulin resistance (reviewed in [1]), might be caused by increased adipose tissue lipolysis and NEFA secretion, increased hepatic VLDL triglyceride (TG) synthesis and secretion, and reduced peripheral NEFA uptake. The cellular uptake of long-chain NEFAs is facilitated by membrane-associated proteins, including fatty acid translocase (FAT/CD36) [2], and 
the fatty acid transport proteins (FATPs) [3]. The FATPs constitute a highly homologous family of proteins (FATP1-6 in man) with distinct expression patterns [4], of which FATP1 and FATP4 are believed to be the main FATPs in adipose tissue. The mechanism of protein-mediated NEFA import is not entirely known, but has been suggested to include vectorial acylation and metabolic trapping of NEFAs via the intrinsic acyl-CoA synthase (ACS) activity of the FATPs [5], possibly in concert with ACS1 activity [6]. Intracellularly, cytosolic fatty-acid-binding proteins (FABPs), which include FABP4/adipocyte lipid-binding protein (ALBP)/aP2 and FABP5/keratinocyte lipidbinding protein (KLBP)/mal1 in adipose tissue [7], are believed to sequester and direct NEFAs and their derivatives into different metabolic pathways.

Several of the above adipose tissue fatty-acidhandling proteins are subject to similar transcriptional $[8,9]$ and post-translational $[10,11]$ regulation, and some co-localise in subcellular compartments $[6,12]$, facts which suggest that fatty-acid-handling protein activity is coordinated. Transcriptional regulators of fatty-acid-handling proteins include molecular entities that are modulated in obesity and insulin resistance, e.g. potential peroxisome proliferator-activated receptor (PPAR)-ligands [8, 13], insulin [14], native and oxidised lipoproteins [15], as well as inflammatory mediators and cytokines [16]. Interestingly, insulin down-regulates adipocyte FATP1 mRNA levels [14] but induces translocation of FATP1 from intracellular pools to the plasma membrane [11], possibly up-regulating protein activity in ways reminiscent of CD36 [10] and GLUT4 [17] translocation in response to insulin. The importance of protein-mediated trans-membrane and intracellular fatty acid transport for lipid and carbohydrate homeostasis has been demonstrated in animal models and by in vitro studies $[18,19,20$, 21]. Fatty-acid-handling protein expression in human tissues in relation to obesity and insulin resistance is poorly investigated, and further studies are clearly warranted. Previous studies of human adipose tissue have shown ALBP and KLBP expression to be increased after weight loss in obese subjects and CD36 expression to be decreased [22]. Moreover, lipodystrophy (which is strongly related to insulin resistance) in HIV-positive patients undergoing highly active antiretroviral therapy was associated with reduced adipose tissue ACS1 expression [23]. In addition, FATP1 mRNA levels in skeletal muscle were negatively correlated with BMI in middle-aged women (but not in men), whereas no such correlation was observed for adipose tissue FATP1 expression, which was analysed in men only [24]. To our knowledge, no studies have shown that FATP4 expression is related to measures of human obesity or insulin resistance.

In general, cross-sectional studies of human disease markers rarely permit unequivocal distinction between genetic versus environmental and life-style effects on the investigated variables. In order to enable such distinctions, the study of monozygotic (MZ) twins discordant for markers of disease is clearly advantageous [25]. In the current study, $17 \mathrm{MZ}$ twin-pairs were recruited specifically because they exhibited a range of intra-pair differences in BMI. Recruitment followed screening of $658 \mathrm{MZ}$ twin-pairs in the FinnTwin 16 cohort, for the purpose of investigating the expression of fatty-acid-handling proteins in adipose tissue in relation to obesity and insulin resistance, allowing the possibility to distinguish between influences of genetic and non-genetic factors on the studied parameters.

\section{Subjects and methods}

Subjects. In order to investigate fatty-acid-handling protein gene expression in adipose tissue in relation to measures of obesity and insulin resistance, with or without the influence of genetic background, MZ twin-pairs with a range of intra-pair differences in BMI were recruited to the study. The study participants were recruited from FinnTwin16, a population-based, longitudinal study including virtually all Finnish twins born between 1975 and 1979 and identified in the Finnish national population registry [26]. Questionnaires were initially sent to 16 -year-old twins, resulting in 5661 responses. The response rates were $88 \%$ and $93 \%$ for boys and girls respectively. All respondent twins were surveyed again at 17 and 18.5 years of age, and as young adults.

Based on responses to the last follow-up questionnaires between 2000 and 2002, twin-pairs were recruited to the current study. After screening of all MZ twin-pairs $(n=658), 14$ pairs with a BMI difference of at least $4 \mathrm{~kg} / \mathrm{m}^{2}$ were identified. Ten such pairs with discordant BMI values participated in the clinical studies. In addition, nine MZ pairs who were seemingly concordant for BMI were studied. Analyses of mRNA expression in subcutaneous fat were performed for nine discordant and eight concordant (four overweight, four with normal weight) $\mathrm{MZ}$ twin-pairs.

All participants gave their informed consent to participate in the study. All were considered healthy (except for obesity) and were not taking medication (except contraceptives) and were also not pregnant, these assessments being based on clinical examination by a physician and a structured psychiatric interview for major psychiatric disorders including depression, eating disorders and substance abuse. The study was approved by the Finnish and Swedish local ethics committees.

Study design. All patients were studied after an overnight fast. Blood samples were taken for measurement of plasma glucose concentrations by the glucose oxidase method using a Beckman Glucose Analyser II (Beckman Instruments, Fullerton, Calif., USA) and serum TG, total and HDL cholesterol concentrations with the respective enzymatic kits (Roche Diagnostics, Branchburg, N.J., USA). LDL cholesterol concentrations were calculated using the Friedewald formula. Whole-body insulin sensitivity (M-value) was assessed with a euglycaemic-hyperinsulinaemic clamp procedure, with measurements of base-line serum insulin (Phadeseph Insulin RIA, Pharmacia and Upjohn Diagnostics, Uppsala, Sweden) and NEFA concentrations (analysed at 0, 30, 60, 90 and 120 minutes) by a fluorometric method [27]. Body fat was measured by dual-energy x-ray absorptiometry as described [28]. Subcutaneous and intra-abdominal fat was measured by magnetic 
Table 1. Physical and biochemical characteristics and specific subcutaneous adipose tissue mRNA expression of the monozygotic (MZ) twins, and intra-pair differences (heavier-leaner) ${ }^{\mathrm{a}}$ in discordant and concordant pairs

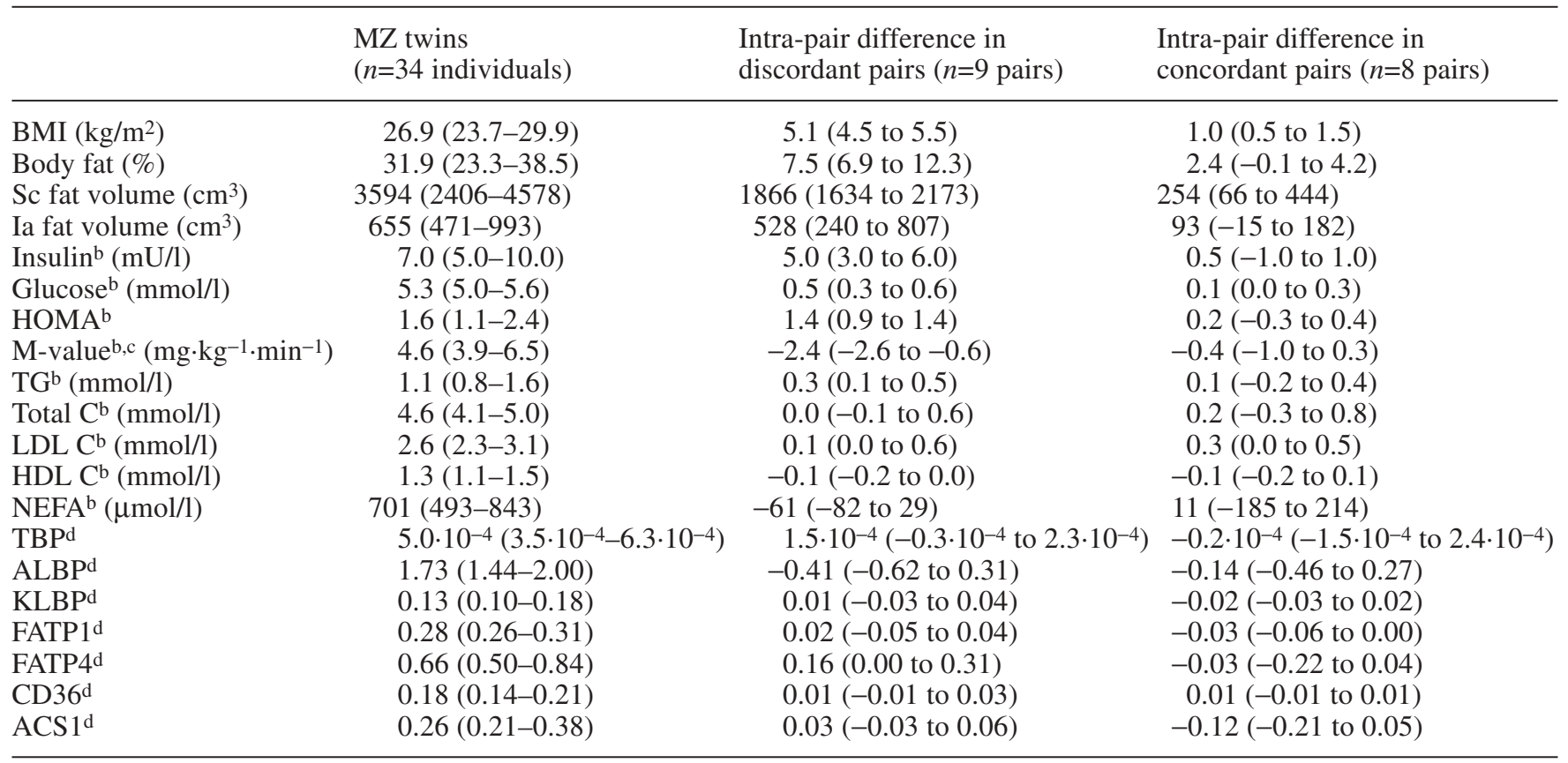

Data are given as median values with inter-quartile range (25th-75th percentile) in parentheses. ${ }^{\text {a }}$ Based on BMI. ${ }^{\mathrm{b}}$ Measured after an overnight fast. ${ }^{c}$ Whole-body insulin sensitivity measured under euglycaemic-hyperinsulinaemic conditions. ${ }^{d}$ mRNA expression levels are given in arbitrary units and the gene expression investigated is given relative to control

resonance imaging of 16 transaxial scans reaching from $8 \mathrm{~cm}$ above to $8 \mathrm{~cm}$ below the fourth and fifth lumbar interspace as described [23].

Analysis of mRNA expression. Subcutaneous abdominal adipose tissue biopsies taken by needle aspiration under local anaesthesia were homogenised in $2 \mathrm{ml}$ RNA STAT-60 (Tel-Test, Friendswood, Tex., USA) and total RNA was isolated as described [23]. For cDNA synthesis we used $100 \mathrm{ng}$ total RNA with M-MLV reverse transcriptase (Life Technologies, Paisley, UK). We quantified mRNA expression of specific fatty-acidhandling proteins (ALBP, KLBP, FATP1, FATP4, CD36, ACS1) by real-time PCR using the ABI Prism 7000 Sequence Detection System and software (Applied Biosystems, Foster City, Calif., USA) according to the manufacturer's protocol and employing five-point standard curves generated from tenfold dilutions of purified PCR-products. Primer and probe sets for ALBP, KLBP, FATP1, FATP4 and ACS1 were designed using the manufacturer's software and sequences deposited in GenBank (NM001442, NM001444, AX015323, AF055899 and L09229 respectively) [23]. CD36 and TATA-box binding protein (TBP) were measured with pre-developed assays-ondemand (Hs00169627_m1 and Hs00427620_m1 respectively; Applied Biosystems). Results were expressed as arbitrary units related to levels of TBP mRNA expression for normalisation.

Statistical analysis. Intra-pair differences between leaner and heavier co-twins were analysed by paired non-parametric Wilcoxon tests. In twin individuals, Pearson correlations were computed to describe the associations between the variables. The statistical significance of the association was derived from regression analyses, with correction for co-twin clustering
mRNA expression (TATA-box binding protein [TBP]). ACS1, acyl-CoA synthase 1; ALBP, adipocyte lipid-binding protein; C, cholesterol; FATP1, fatty acid transport protein 1; FATP4, fatty acid transport protein 4; HOMA, homeostasis model assessment; Ia, intra-abdominal; KLBP, keratinocyte lipid-binding protein; Sc, subcutaneous; TG, triglyceride

compensating for within-pair relationships of observations. Because the twin subjects are clustered in pairs rather than derived from true population individuals, the error terms, confidence intervals and $p$ values would be biased were this clustering ignored. Skewed variables were logarithmically transformed prior to individual correlation analysis. Spearman rank correlations were used for intra-pair analyses. The analyses were conducted with the Stata software (release 8.0; Stata, College Station, Tex., USA) or the Statview software (SAS Institute, Cary, N.C., USA). The homeostasis model assessment (HOMA) index was calculated as the product of fasting insulin and glucose concentrations divided by 22.5. A $p$ value of 0.05 or less was deemed statistically significant.

\section{Results}

The mean age of the study participants was 25.7 years (23.8-27.4 years). Eight pairs were women and nine pairs were men. Physical and biochemical characteristics and subcutaneous adipose tissue gene expression levels of the study participants are presented in Table 1. In accordance with the selection procedure, both the discordant and the concordant groups showed intra-pair differences in BMI of a similar statistical significance ( $p=0.008$ and $p=0.01$ respectively), but different numerical magnitude. Consistently, in the discordant group, body fat, subcutaneous fat, intraabdominal fat, M-value, and insulin, glucose and HDL 
Table 2. Individual correlations between specific subcutaneous adipose tissue mRNA expression and physical and biochemical variables

\begin{tabular}{|c|c|c|c|c|c|c|}
\hline & \multicolumn{6}{|c|}{ mRNA expression $(n=34)$} \\
\hline & ALBP & KLBP & FATP1 & FATP4 & CD36 & ACS1 \\
\hline Body fat & -0.13 & -0.03 & -0.09 & $0.51^{\mathrm{c}}$ & $0.63^{\mathrm{d}}$ & -0.03 \\
\hline Sc fat volume & -0.07 & 0.05 & -0.06 & $0.62^{\mathrm{e}}$ & $0.71^{\mathrm{e}}$ & 0.07 \\
\hline Ia fat volume & 0.18 & $0.49^{c}$ & -0.25 & $0.30^{\mathrm{b}}$ & 0.14 & 0.15 \\
\hline HOMA & 0.07 & 0.11 & -0.16 & $0.49^{\mathrm{d}}$ & $0.34^{\mathrm{a}}$ & -0.17 \\
\hline M-value & 0.14 & 0.05 & 0.17 & -0.33 & $-0.35^{a}$ & 0.07 \\
\hline $\mathrm{TG}$ & 0.27 & $0.42^{b}$ & -0.17 & -0.13 & -0.02 & -0.04 \\
\hline Total C & 0.25 & 0.30 & -0.02 & $0.34^{c}$ & $0.43^{\mathrm{b}}$ & 0.20 \\
\hline HDL C & -0.03 & -0.15 & $0.36^{\mathrm{a}}$ & -0.12 & 0.13 & 0.02 \\
\hline LDL C & 0.19 & 0.31 & -0.10 & $0.48^{\mathrm{c}}$ & $0.46^{\mathrm{b}}$ & 0.17 \\
\hline
\end{tabular}

Pearson correlation coefficients and significance levels corrected for twin clustering stated. For units, see Table 1. a $p=0.06-0.08, \quad{ }^{\mathrm{b}} p \leq 0.05, \quad{ }^{\mathrm{c}} p \leq 0.01, \quad{ }^{\mathrm{d}} p \leq 0.001, \quad{ }^{\mathrm{e}} p \leq 0.0001$. ACS1, acyl-CoA synthase 1; ALBP, adipocyte lipid-binding protein; C, cholesterol; FATP1, fatty acid transport protein 1; FATP4, fatty acid transport protein 4; HOMA, homeostasis model assessment; Ia, intra-abdominal; KLBP, keratinocyte lipid-binding protein; Sc, subcutaneous; TG, triglyceride

Table 3. Pairwise correlations between intra-pair differences $(\Delta)$ in specific subcutaneous adipose tissue mRNA expression and intra-pair differences $(\Delta)$ in physical and biochemical variables

\begin{tabular}{|c|c|c|c|c|c|c|}
\hline & \multicolumn{6}{|c|}{ Intra-pair difference in mRNA expression $(n=17)$} \\
\hline & $\triangle \mathrm{ALBP}$ & $\triangle \mathrm{KLBP}$ & $\Delta \mathrm{FATP} 1$ & $\Delta$ FATP4 & $\Delta \mathrm{CD} 36$ & $\triangle \mathrm{ACS} 1$ \\
\hline$\Delta$ Body fat & 0.09 & 0.21 & 0.13 & $0.59^{\mathrm{c}}$ & -0.03 & 0.23 \\
\hline$\Delta \mathrm{Sc}$ fat volume & -0.05 & -0.06 & -0.003 & $0.51^{\mathrm{b}}$ & 0.04 & 0.22 \\
\hline$\Delta \mathrm{Ia}$ fat volume & -0.24 & 0.11 & 0.09 & $0.48^{\mathrm{b}}$ & -0.21 & 0.29 \\
\hline$\triangle \mathrm{HOMA}$ & 0.24 & 0.13 & 0.13 & 0.17 & 0.10 & -0.01 \\
\hline$\Delta \mathrm{M}$-value & -0.23 & -0.003 & 0.13 & -0.32 & 0.11 & 0.20 \\
\hline$\Delta \mathrm{TG}$ & -0.12 & $-0.46^{\mathrm{a}}$ & -0.22 & -0.07 & 0.20 & 0.06 \\
\hline$\Delta$ Total C & -0.01 & $-0.43^{a}$ & -0.28 & 0.02 & 0.26 & -0.15 \\
\hline$\Delta \mathrm{HDL} \mathrm{C}$ & 0.03 & -0.15 & 0.03 & -0.27 & $0.56^{\mathrm{b}}$ & 0.005 \\
\hline$\Delta \mathrm{LDL} \mathrm{C}$ & 0.001 & -0.37 & -0.23 & 0.17 & -0.10 & -0.24 \\
\hline
\end{tabular}

Spearman correlation coefficients and significance levels for heavier-leaner co-twin differences stated. For units, see Table 1. ${ }^{\mathrm{a}} p=0.06-0.08,{ }^{\mathrm{b}} p \leq 0.05,{ }^{\mathrm{c}} p \leq 0.01$. ACS1, acyl-CoA synthase 1; ALBP, adipocyte lipid-binding protein; C, choles-

cholesterol concentrations were also significantly different between heavier and leaner twins, whereas in the concordant group no such differences in other anthropometric or biochemical variables were observed. However, FATP1 expression levels were significantly different between heavier and leaner twins in the concordant group $(p=0.05)$. Control TBP mRNA expression levels were not significantly correlated with BMI in either individual $(r=-0.18, p=0.40$, $n=34$ with correction for co-twin clustering) or intrapair analyses $(r=-0.07, p=0.79, n=17)$. terol; FATP1, fatty acid transport protein 1; FATP4, fatty acid transport protein 4; HOMA, homeostasis model assessment; Ia, intra-abdominal; KLBP, keratinocyte lipid-binding protein; Sc, subcutaneous; TG, triglyceride

In individual correlation analysis (Table 2), influenced by genetic, environmental and life-style factors, FATP4 and CD36 expression levels in subcutaneous adipose tissue were found to be positively correlated with BMI, body fat and subcutaneous fat (Fig. 1, 2). FATP4 and KLBP expression levels were positively correlated with intra-abdominal fat. FATP4 expression correlated positively with insulin concentrations and the HOMA index. CD36 expression also correlated with insulin concentrations, and (border-line significance) with the HOMA index and M-value. Further- 
a
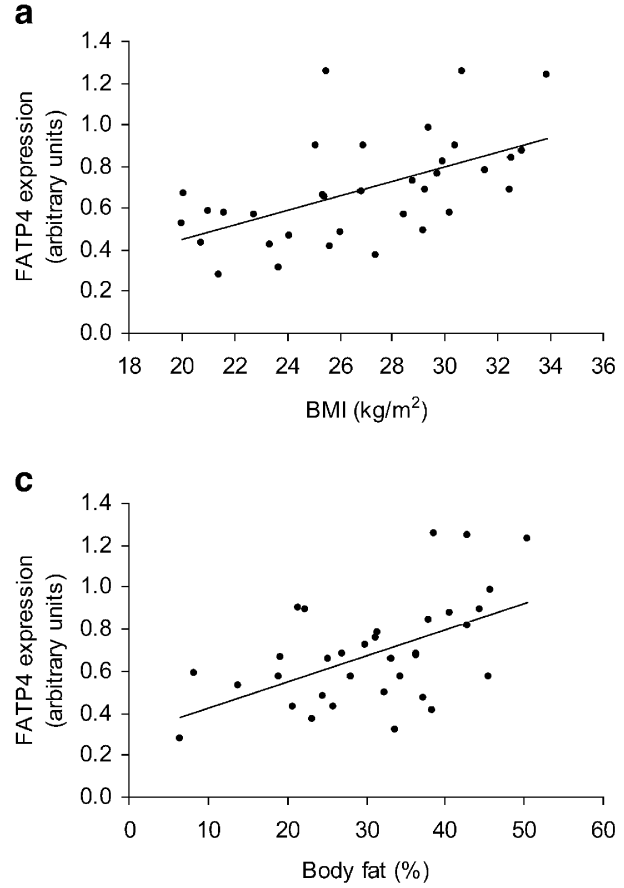

Fig. 1. Scatter plots of the relation between FATP4 mRNA expression in subcutaneous adipose tissue and BMI (a) and body fat (c) in individuals, and of the relation between intrapair differences in subcutaneous adipose tissue FATP4 mRNA
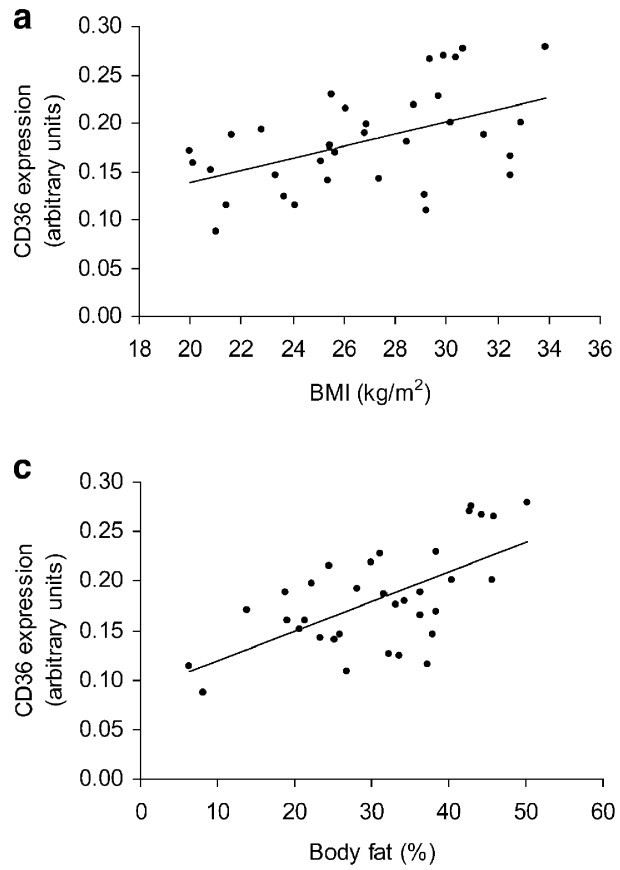

Fig. 2. Scatter plots of the relation between CD36 mRNA expression in subcutaneous adipose tissue and BMI (a) and body fat (c) in individuals, and of the relation between intrapair differences in subcutaneous adipose tissue CD36 mRNA
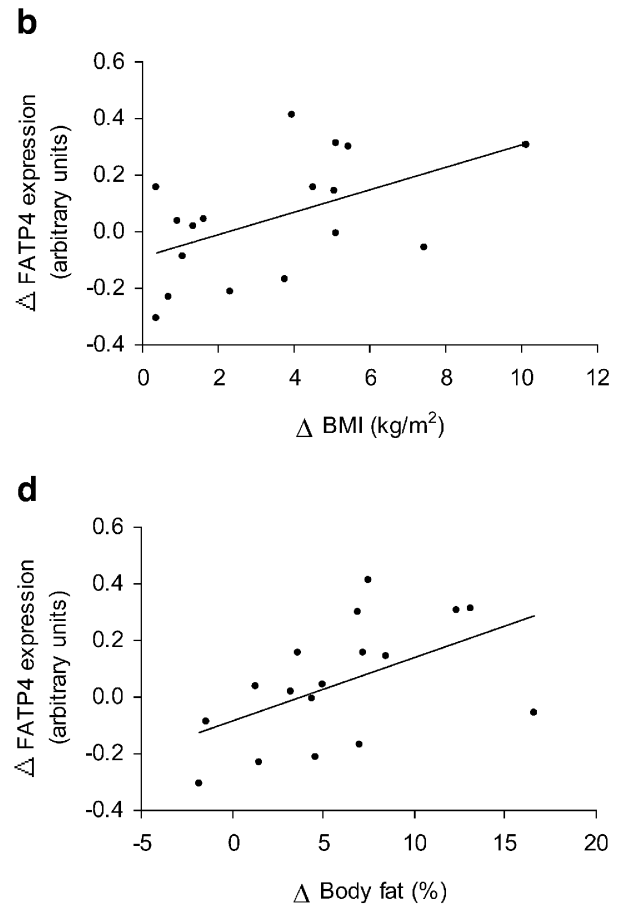

expression and intra-pair differences in BMI (b) and body fat (d). Regression lines are shown. For statistics, see Table 2 (a, c) and Table $3(\mathbf{b}, \mathbf{d})$
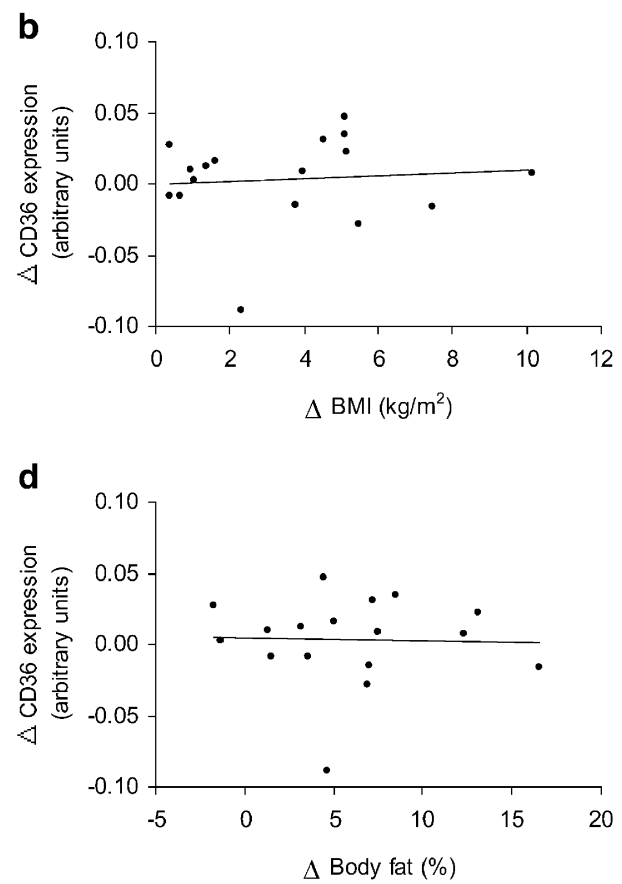

expression and intra-pair differences in BMI (b) and body fat (d). Regression lines are shown. For statistics, see Table 2 (a, c) and Table $3(\mathbf{b}, \mathbf{d})$ 
more, FATP4 and CD36 expression levels were positively correlated with total and LDL cholesterol concentrations, and KLBP expression with TG concentrations. FATP1 expression had a positive correlation with HDL cholesterol (border-line significance). ACS1 was negatively correlated and FATP1 expression was positively correlated (border-line significance) with base-line (fasting) NEFA concentrations before a hyperinsulinaemic-euglycaemic clamp. However, after 30 and $60 \mathrm{~min}$ of the clamp respectively, the correlations of ACS1 and FATP1 expression with NEFA concentrations were no longer significant. Given the similar observations above with regard to FATP4 and CD36 expression, the correlation between the expression levels of these genes was investigated and found to be highly significant $(r=0.66, p=0.0001)$.

In the analysis of intra-pair differences (Table 3), independent of genetic factors, FATP4 expression levels were positively correlated with measures of adiposity (BMI, body fat, subcutaneous fat and intra-abdominal fat; Fig. 1), whereas CD36 expression levels were not (Fig. 2). CD36 expression was positively correlated with HDL cholesterol concentrations. FATP1 and ALBP expression (the latter with borderline significance) were positively correlated with NEFA concentrations. KLBP expression levels were negatively correlated with TG and total cholesterol concentrations (border-line significance). In contrast to the individual correlation analysis, no significant correlation between intra-pair differences in FATP4 and CD36 expression levels was observed.

\section{Discussion}

The current study was designed to allow the investigation of subcutaneous adipose tissue gene expression in relation to obesity and insulin resistance, with or without the influence of the genetic background. To this end, MZ twins exhibiting a range of within-pair differences in detailed and extensive measures of obesity, fat distribution and insulin resistance were recruited to the study. Two modes of statistical evaluation were implemented: (i) individual correlation analyses corrected for co-twin clustering to investigate gene expression in relation to obesity and insulin resistance caused by genetic and non-genetic factors; and most importantly (ii) intra-pair analyses to investigate gene expression in relation to acquired obesity and insulin resistance, independent of genetic factors.

Expression levels of several fatty-acid-handling proteins were related to markers of obesity and insulin resistance caused by the combined effects of genes, environment and life-style, according to the individual analysis. FATP4 and CD36 expression levels were positively correlated with measures of obesity (BMI, body fat, subcutaneous and intra-abdominal fat), and consistently also with insulin concentrations and the
HOMA index. This is consistent with observations that up-regulation of adipocyte fatty acid transporter mRNA levels and fatty acid uptake occur early in rat models of genetic obesity [29]. Also, adipose-tissuederived giant vesicles (in which fatty acid uptake is divorced from cellular fatty acid metabolism) from obese rats exhibited higher fatty acid transporter expression and fatty acid uptake than vesicles from lean littermates [30]. However, in manifest human obesity and insulin resistance, adipose tissue fatty acid uptake and buffering are impaired [1]. The idea that FATP4 plays a role in the development of human obesity and insulin resistance is supported by the association of the FATP4 Gly209Ser polymorphism with variations in BMI, insulin concentrations and HOMA index in healthy middle-aged men [31]. In addition, both FATP4 and CD36 expression correlated positively in the present study, with total and LDL cholesterol concentrations. It has been suggested that CD36, being a lipoprotein scavenger receptor, plays a role in cholesterol metabolism [32]. FATP4, on the other hand, has to date not been directly implicated in cholesterol metabolism.

KLBP expression correlated with intra-abdominal fat volume and TG concentrations. In mice, KLBP deficiency was associated with reduced subcutaneous fat mass and TG concentrations [33], which seems consistent with the current findings. In this study, no significant correlations were observed regarding expression levels of ALBP, the major FABP in adipose tissue. This possibly suggests that KLBP expression is more stringently regulated than ALBP by the parameters investigated in this study. Such differences in the regulation of ALBP and KLBP expression might explain the differences in the ALBP : KLBP ratio observed in lean versus obese subjects [22] and in subcutaneous versus intra-abdominal fat depots [34].

Both ACS1 and FATP1 (as well as CD36 and FATP4) promote NEFA uptake and/or acyl-CoA formation $[5,6]$, thereby possibly counteracting adipose tissue lipolytic NEFA secretion. Consistently in the present study, ACS1 expression correlated negatively with fasting NEFA concentrations. The trend towards a positive correlation between FATP1 expression and fasting NEFA concentrations could result from high FATP1 expression being a consequence rather than a cause of the high NEFA concentrations.

Analysis of gene expression related to acquired obesity and insulin resistance independent of genetic background showed that FATP4 expression was upregulated in acquired obesity, arguing that the influence of environmental and life-style factors on FATP4 expression are important for the development of obesity. FATP4 might thus be an attractive target for pharmaceutical prevention or treatment of obesity. In contrast, no significant (numerically almost zero) correlations between acquired obesity and CD36 expression were observed, suggesting that the correlations 
between CD36 expression and obesity in the individual analysis were due mainly to genetic factors. In the individual analysis, the expression levels of FATP4 and CD36 correlated strongly, which could indicate that these gene products share some regulatory properties. However, no such correlation was observed in the analysis of intra-pair differences, suggesting qualitative and/or quantitative differences in transcriptional regulation of FATP4 and CD36 by obesity-related environmental factors. Furthermore, the current data suggest that in acquired obesity, CD36 and FATP1 expression are related to HDL cholesterol and NEFA concentrations respectively.

The current study design is particularly valuable because it permits the identification of parameters related to acquired obesity and insulin resistance. However, it should be noted that this study only identifies specific subcutaneous adipose tissue gene expression related to the development of obesity and insulin resistance, without characterising causal relationships. Moreover, the mRNA data may not accurately reflect protein levels or protein activity, especially as this activity could be dependent on sub-cellular protein localisation. However, commercially available antibodies have not allowed us unambiguous quantification of adipose tissue fatty-acid-handling protein expression (e.g. FATP4) by western blot analysis. In addition, several of the investigated fatty-acidhandling proteins exhibit tissue-specific expression patterns (e.g. FATPs and FABPs), and this may reflect physiological differences in fatty acid handling between different tissues at a given nutritional state (for example, adipose tissue TG hydrolysis and hepatic TG synthesis occurring concomitantly in the fasting state). The current observations in subcutaneous adipose tissue should not therefore be extrapolated to other tissues. Instead, further studies are necessary to elucidate the physiological role(s) of fatty-acid-handling proteins in different tissues.

In summary, we have provided evidence that expression levels of specific fatty-acid-handling proteins in subcutaneous adipose tissue are related to measures of genetic and acquired obesity and insulin resistance in young people. In particular, we found in individual correlation analyses that FATP4 and CD36 are related to measures of obesity and insulin resistance, while intra-pair correlations revealed that FATP4 expression was significantly up-regulated in acquired obesity. These findings further suggest that facilitated fatty acid transport in subcutaneous adipose tissue is a concept of physiological and pathological relevance in man.

Acknowledgements. This work was supported by the Swedish Medical Research Council (project 8691), the Torsten and Ragnar Söderberg Foundation, the Swedish Heart-Lung Foundation, the Swedish Institute, the Swedish National Network and Graduate School for Cardiovascular Research, the Profes- sor Nanna Svartz Foundation, the Ake Wiberg Foundation, the Nilsson-Ehle Foundation, the Fredrik and Ingrid Thuring Foundation, the Gamla Tjänarinnor Foundation, the Swedish Society of Medicine, the Academy of Finland (44069, 201461), the USPHS National Institute of Alcohol Abuse and Alcoholism (AA08315, AA12502), the European Union Fifth Framework Program (QLRT-1999-00916, QLG2-CT-200201254), Helsinki University Central Hospital grants, the Finnish Medical Association, the Finnish Cultural Foundation, the Yrjö Jahnsson Foundation and the Ahokas Foundation. The authors wish to thank Erjastiina Heikkinen, Katja Tuominen and Maarit Toivonen for excellent assistance. They also thank all volunteers. K. Gertow and K. H. Pietiläinen contributed equally to this work.

\section{References}

1. Frayn KN (2002) Adipose tissue as a buffer for daily lipid flux. Diabetologia 45:1201-1210

2. Abumrad NA, el-Maghrabi MR, Amri EZ, Lopez E, Grimaldi PA (1993) Cloning of a rat adipocyte membrane protein implicated in binding or transport of long-chain fatty acids that is induced during preadipocyte differentiation. Homology with human CD36. J Biol Chem 268: 17665-17668

3. Schaffer JE, Lodish HF (1994) Expression cloning and characterization of a novel adipocyte long chain fatty acid transport protein. Cell 79:427-436

4. Hirsch D, Stahl A, Lodish HF (1998) A family of fatty acid transporters conserved from mycobacterium to man. Proc Natl Acad Sci USA 95:8625-8629

5. Coe NR, Smith AJ, Frohnert BI, Watkins PA, Bernlohr DA (1999) The fatty acid transport protein (FATP1) is a very long chain acyl-CoA synthetase. J Biol Chem 274:3630036304

6. Gargiulo CE, Stuhlsatz-Krouper SM, Schaffer JE (1999) Localization of adipocyte long-chain fatty acyl-CoA synthetase at the plasma membrane. J Lipid Res 40:881-892

7. Coe NR, Bernlohr DA (1998) Physiological properties and functions of intracellular fatty acid- binding proteins. Biochim Biophys Acta 1391:287-306

8. Martin G, Schoonjans K, Lefebvre AM, Staels B, Auwerx J (1997) Coordinate regulation of the expression of the fatty acid transport protein and acyl-CoA synthetase genes by PPARalpha and PPARgamma activators. J Biol Chem 272:28210-28217

9. Van Nieuwenhoven FA, Willemsen PH, Van der Vusse GJ, Glatz JF (1999) Co-expression in rat heart and skeletal muscle of four genes coding for proteins implicated in long-chain fatty acid uptake. Int J Biochem Cell Biol 31:489-498

10. Luiken JJ, Dyck DJ, Han XX et al. (2002) Insulin induces the translocation of the fatty acid transporter FAT/CD36 to the plasma membrane. Am J Physiol Endocrinol Metab 282:E491-E495

11. Stahl A, Evans JG, Pattel S, Hirsch D, Lodish HF (2002) Insulin causes fatty acid transport protein translocation and enhanced fatty acid uptake in adipocytes. Dev Cell 2:477-488

12. Gimeno RE, Ortegon AM, Patel S et al. (2003) Characterization of a heart-specific fatty acid transport protein. J Biol Chem 278:16039-16044

13. Van der Lee KA, Vork MM, De Vries JE et al. (2000) Long-chain fatty acid-induced changes in gene expression in neonatal cardiac myocytes. J Lipid Res 41:41-47 
14. Man MZ, Hui TY, Schaffer JE, Lodish HF, Bernlohr DA (1996) Regulation of the murine adipocyte fatty acid transporter gene by insulin. Mol Endocrinol 10:1021-1028

15. Han J, Hajjar DP, Febbraio M, Nicholson AC (1997) Native and modified low density lipoproteins increase the functional expression of the macrophage class B scavenger receptor, CD36. J Biol Chem 272:21654-21659

16. Memon RA, Feingold KR, Moser AH, Fuller J, Grunfeld C (1998) Regulation of fatty acid transport protein and fatty acid translocase mRNA levels by endotoxin and cytokines. Am J Physiol 274:E210-E217

17. Suzuki K, Kono T (1980) Evidence that insulin causes translocation of glucose transport activity to the plasma membrane from an intracellular storage site. Proc Natl Acad Sci USA 77:2542-2545

18. Ibrahimi A, Bonen A, Blinn WD et al. (1999) Musclespecific overexpression of FAT/CD36 enhances fatty acid oxidation by contracting muscle, reduces plasma triglycerides and fatty acids, and increases plasma glucose and insulin. J Biol Chem 274:26761-26766

19. Febbraio M, Abumrad NA, Hajjar DP et al. (1999) A null mutation in murine CD36 reveals an important role in fatty acid and lipoprotein metabolism. J Biol Chem 274:1905519062

20. Stahl A, Gimeno RE, Tartaglia LA, Lodish HF (2001) Fatty acid transport proteins: a current view of a growing family. Trends Endocrinol Metab 12:266-273

21. Boord JB, Fazio S, Linton MF (2002) Cytoplasmic fatty acid-binding proteins: emerging roles in metabolism and atherosclerosis. Curr Opin Lipidol 13:141-147

22. Fisher RM, Hoffstedt J, Hotamisligil GS, Thorne A, Ryden M (2002) Effects of obesity and weight loss on the expression of proteins involved in fatty acid metabolism in human adipose tissue. Int $\mathrm{J}$ Obes Relat Metab Disord 26:1379-1385

23. Kannisto K, Sutinen J, Korsheninnikova E et al. (2003) Expression of adipogenic transcription factors, peroxisome proliferator-activated receptor gamma co-activator 1 , IL-6 and CD45 in subcutaneous adipose tissue in lipodystrophy associated with highly active antiretroviral therapy. AIDS 17:1753-1762
24. Binnert C, Koistinen HA, Martin G et al. (2000) Fatty acid transport protein-1 mRNA expression in skeletal muscle and in adipose tissue in humans. Am J Physiol Endocrinol Metab 279:E1072-E1079

25. Boomsma D, Busjahn A, Peltonen L (2002) Classical twin studies and beyond. Nat Rev Genet 3:872-882

26. Kaprio J, Pulkkinen L, Rose RJ (2002) Genetic and environmental factors in health-related behaviors: studies on Finnish twins and twin families. Twin Res 5:366371

27. Miles J, Glasscock R, Aikens J, Gerich J, Haymond M (1983) A microfluorometric method for the determination of free fatty acids in plasma. J Lipid Res 24:96-99

28. Mazess RB, Barden HS, Bisek JP, Hanson J (1990) Dualenergy $\mathrm{x}$-ray absorptiometry for total-body and regional bone-mineral and soft-tissue composition. Am J Clin Nutr 51:1106-1112

29. Berk PD, Zhou SL, Kiang CL et al. (1997) Uptake of long chain free fatty acids is selectively up-regulated in adipocytes of Zucker rats with genetic obesity and noninsulin-dependent diabetes mellitus. J Biol Chem 272:88308835

30. Luiken JJ, Arumugam Y, Dyck DJ et al. (2001) Increased rates of fatty acid uptake and plasmalemmal fatty acid transporters in obese Zucker rats. J Biol Chem 276:4056740573

31. Gertow K, Bellanda M, Eriksson P et al. (2004) Genetic and structural evaluation of fatty acid transport protein-4 in relation to markers of the insulin resistance syndrome. J Clin Endocrinol Metab 89:392-399

32. Endemann G, Stanton LW, Madden KS et al. (1993) CD36 is a receptor for oxidized low density lipoprotein. J Biol Chem 268:11811-11816

33. Maeda K, Uysal KT, Makowski L et al. (2003) Role of the fatty acid binding protein mal1 in obesity and insulin resistance. Diabetes 52:300-307

34. Fisher RM, Eriksson P, Hoffstedt J et al. (2001) Fatty acid binding protein expression in different adipose tissue depots from lean and obese individuals. Diabetologia 44:1268-1273 